\title{
Bivariate Empirical Mode Decomposition for Unbalanced Real-World Signals
}

\author{
Alireza Ahrabian, Naveed Ur Rehman, and Danilo Mandic, Fellow, IEEE
}

\begin{abstract}
The bivariate empirical mode decomposition (BEMD) algorithm employs uniform sampling on a circle to perform projections in multiple directions, in order to calculate the local mean of a bivariate signal. However, this approach is adequate only for equal powers in both the data channels within a bivariate signal, and results in suboptimal performance for data channels exhibiting power imbalance, a typical case in practice. To that end, we exploit second-order bivariate statistical properties to introduce a nonuniform sampling scheme for data adaptive selection of the projection directions. In this way, the resulting nonuniformly sampled BEMD (NS-BEMD) algorithm provides a more accurate time-frequency representation of bivariate data than standard BEMD, for the same number of projections. The advantages of the proposed approach are demonstrated in case studies on BEMD for correlated data channels, selection of optimal noise power in noise-assisted BEMD, and for speed estimation using Doppler radar.
\end{abstract}

Index Terms-Bivariate empirical mode decomposition, Hilbert-Huang transform, nonuniform sampling.

\section{INTRODUCTION}

$\mathbf{T}$ HE EMPIRICAL mode decomposition (EMD) is a data driven method for the analysis of nonstationary data [1], which operates by decomposing a signal into a number of oscillatory modes, termed intrinsic mode functions (IMFs). These IMFs are defined such that the application of the Hilbert transform yields much more localized time-frequency estimates, than those obtained via standard techniques, such as short-time Fourier transform and wavelets. The power of EMD comes from its fully data-driven nature, it makes no a priori assumptions on input data, making it highly suitable for applications involving non-stationary signals.

The EMD in its original form operates on univariate signals and thus cannot handle cross-channel properties in multivariate data. On the other hand, recent advances in sensor technology and data acquisition tools have made possible routine recording from multiple data channels (multivariate signals), for which EMD based analysis tools are only emerging. For the case of bivariate (or complex valued) data, several extensions of EMD

Manuscript received October 30, 2012; revised December 28, 2012; accepted January 15, 2013. Date of publication January 23, 2013; date of current version February 01,2013 . The associate editor coordinating the review of this manuscript and approving it for publication was Prof. Petros T. Boufounos.

The authors are with the Department of Electrical and Electronic Engineering, Imperial College London, London SW7 2AZ, U.K. and also with the Department of Electrical Engineering, COMSATS Institute of Information Technology, Islamabad, Pakistan (e-mail: alireza.ahrabian06@imperial.ac.uk; d.mandic@imperial.ac.uk; nrehman.comsats@gmail.com).

Color versions of one or more of the figures in this paper are available online at $\mathrm{http}: / /$ ieeexplore.ieee.org.

Digital Object Identifier 10.1109/LSP.2013.2242062 have been recently proposed: 1) Complex EMD by Tanaka and Mandic [2], 2) Rotation- invariant EMD by Altaf et al. [3], and 3) bivariate EMD by Rilling et al. [4].

Complex EMD (CEMD) applies standard univariate EMD separately to the real and imaginary data channels, thus not guaranteeing the same number of IMFs across data channels, which is a major requirement in real-world applications. The rotation-invariant EMD (RI-EMD) is a fully bivariate (complex) extension of EMD, which operates by taking projections of the bivariate input signal along two directions in the complex plane to compute the local mean. The bivariate signal envelopes are calculated by interpolating the envelopes of those univariate (real-valued) projections, and the local mean of a complex signal is determined by taking the mean of the envelopes. Although the RI-EMD gives the same number of IMFs for both the signal components, it is not well-equipped to deal with fast signal dynamics due to a low number of signal projections, which limits its practical usefulness.

To alleviate these problems, Rilling et al. [4] developed the bivariate EMD (BEMD) algorithm, which takes multiple (univariate) projections of a complex (bivariate) signal to determine the local mean. Unlike RI-EMD, the multiple projection directions within BEMD can sample the whole complex (bivariate) plane, enhancing accuracy and making it much better suited for signals with fast changing dynamics than RI-EMD. More recently, other algorithms such as 2T-EMD [5] have been proposed, in order to balance between accuracy and computational complexity.

Owing to its accurate estimation of the local mean, the BEMD has proven successful in bivariate non-stationary signal analysis, particularly in data fusion applications [6]. It, however, employs static (fixed) distribution of direction vectors (along polar coordinates in 2D) thus not catering for second order properties of bivariate data, such as power discrepancies between the data channels. This affects both the accuracy and the computational complexity of the algorithm. Moreover, noise-assisted extensions of multivariate EMD (NA-MEMD) [7], [8] are also emerging as an effective way to calculate univariate EMD, and critically depend on the ratio of powers in the data channels, which further highlights the need for a data adaptive sampling procedure in BEMD.

We here introduce a nonuniform projection scheme for calculating the local mean of bivariate data, whereby the direction vectors for projections are constructed so as to account for second-order statistics of data channels within a bivariate signal. This makes it possible to cater for both inherent correlations and power variations present in the input data. The nature of the bivariate or complex signal is first examined by computing the circularity quotient, from which parameters for nonuniform data-adaptive projections are selected. The proposed strategy 
is shown to provide, on the average, more localized time-frequency estimates for unbalanced data, and better accuracy for the same number of projections, when compared to the conventional BEMD algorithm. Simulations on both synthetic and real-world data support the approach.

\section{Bivariate Empirical Mode Decomposition}

The BEMD algorithm operates by first projecting an input bivariate signal in $N$ uniformly spaced directions along a unit circle, as shown in Fig. 1 (left). The extrema points of such multiple real univariate projections are calculated separately, while the interpolation of such extrema results in bivariate envelopes, one for for each direction. The sifting process is thus carried out according to conventional EMD. Algorithm 1 shows the steps required for calculating the local mean of a bivariate signal in BEMD [4].

Algorithm 1: Bivariate Empirical Mode Decomposition

1) Given a set of directions $\phi_{k}=(2 \pi k / N)$, where $k=1, \ldots, N$ project the complex valued signal $s(t)$ along the directions $\phi_{k}$ :

$$
p_{\phi_{k}}(t)=\operatorname{Re}\left(e^{j \phi_{k}} s(t)\right) .
$$

2) Extract the locations $t_{i}^{k}$ of the extrema points of $p_{\phi_{k}}(t)$.

3) Interpolate the set $\left(t_{i}^{k}, s\left(t_{i}^{k}\right)\right)$ to obtain the envelope curve $e_{\phi_{k}}(t)$ in the direction $\phi_{k}$.

4) Compute the mean of all envelope curves:

$$
m(t)=\frac{1}{N} \sum_{k} e_{\phi_{k}}(t) .
$$

5) Subtract $m(t)$ from the input signal in order to obtain the oscillatory component $d(t)$.

\section{NONUNIFORMLY SAMPLED BEMD}

The proposed algorithm performs nonuniform sampling by taking signal projections along the directions determined by the statistical nature of the input: inter-channel correlation and power discrepancy between data channels. For convenience, consider a complex-valued signal $z=x+i y$. Motivated by recent advances in complex valued signal processing, we employ the circularity quotient as a metric to determine the correlation pattern of a signal, yielding the direction of principal importance in the scatter plot of the data. Subsequently, concepts from elliptical geometry are adopted to map the uniform projection vector direction set to a nonuniform direction set tilted along the principal direction (shown in Fig. 1 (right)), thus performing the local mean estimation that adapts to the second order statistics of the signal in hand.

The starting point of the proposed nonuniformly sampled BEMD is to determine the 'principal' direction vector (angle) in a 2D space, corresponding to significant channel correlation and/or dominant channel power. This is achieved by first estimating the circularity quotient [9], given by:

$$
\rho=\frac{P}{C}
$$

where $P=E\left\{z z^{T}\right\}$ is the pseudo-covariance, and $C=E\left\{z z^{H}\right\}$ is the covariance, and $E\{\cdot\}$ is the statistical expectation operator. By definition, $\rho$ is a complex number where $|\rho|$ gives the degree of correlation (or power imbalance) between two data channels and $\arg (\sqrt{\rho})$ the direction in 2D along which such correlations exist. The proposed algorithm finds a set of projection vectors distributed according to the principal (importance) direction $\theta$, as opposed to uniform sampling performed by standard BEMD. The direction of principal importance $\theta$ represents the tilt of the major axis $a$ of an ellipse. For correlated data, the degree of correlation is then related to the eccentricity $\epsilon$ of the ellipse. The modulated elliptical representation used in this work is given by [10]

$$
y_{k}=e^{j \theta}\left(a \cos \left(\phi_{k}\right)+j b \sin \left(\phi_{k}\right)\right)
$$

where $a$ and $b$ are respectively the major and minor axis, and $\theta$ denotes the angle between the $x$-axis and the major axis of an ellipse. The relationship between the major and minor axis is defined as the eccentricity 1 ,

$$
\epsilon=\sqrt{\frac{a^{2}-b^{2}}{a^{2}+b^{2}}}
$$

which governs the spread of correlation. For a given bivariate signal, the parameters $\theta$ and $\epsilon$ can be estimated, via the circularity quotient $\rho$, in (3) as

$$
\epsilon=\sqrt{|\rho|} \quad \theta=\arg (\sqrt{\rho}) .
$$

In this way, uniformly distributed projection vectors in BEMD are converted to elliptically distributed points $y_{k}$; see Algorithm 2 for details of the proposed nonuniformly sampled BEMD (NSBEMD) algorithm.

\section{Algorithm 2: Nonuniformly Sampled BEMD (NS-BEMD)}

1) Estimate the channel powers within a bivariate signal and their correlation, to give the eccentricity $\epsilon$ and angle $\theta$ using the circularity quotient in (3) and (6).

2) Given a set of uniformly distributed angles $\phi_{k}=(2 \pi k / N-1), k=0, \ldots, N-1$, calculate the set of elliptically distributed points ${ }^{2}$, using (4), where $a=\sqrt{(1+|\rho|) / 4}, b=\sqrt{(1-|\rho|) / 4}$.

3) Perform projections of the bivariate signal along the directions $\phi_{k}^{\prime}=\arg \left(y_{k}\right)$ where $y_{k}$ is given in (4), and continue as in BEMD.

Fig. 2 shows 16 elliptically distributed directions generated by NS-BEMD for bivariate signals of equal channel powers but with varying inter-channel correlations $\eta$ (for convenience the samples have been projected across a unit circle). As desired, the projection directions are denser around the direction of principal importance, $\theta$, as the channel correlation increases. Fig. 3 shows the 16 points corresponding to the projection directions

\footnotetext{
${ }^{1}$ The eccentricity parameter used in this work corresponds to the eccentricity from [9]. There are, however, other definitions of eccentricity available which can be used within the proposed framework, including the more widely used definition of eccentricity found in [10].

${ }^{2}$ The major and minor axis of the ellipse have been selected such that (5) and the condition $a^{2}+b^{2}=1$ hold [11]
} 


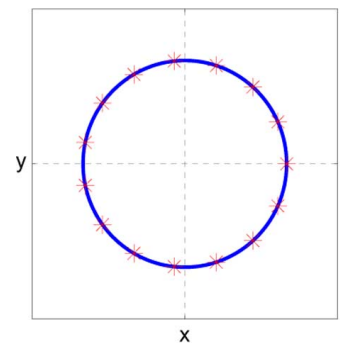

(a)

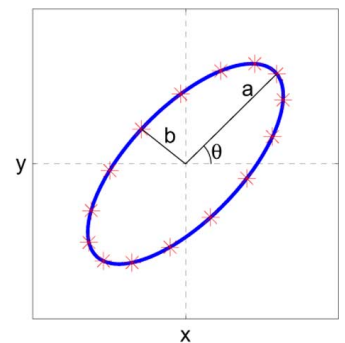

(b)
Fig. 1. Scatter plot for a bivariate signal, $z=x+i y$. (Left) A uniformly sampled unit circle (standard BEMD). (Right) A uniformly sampled ellipse, with higher density of samples along the major axis $a$ (the proposed NS-BEMD). (a)

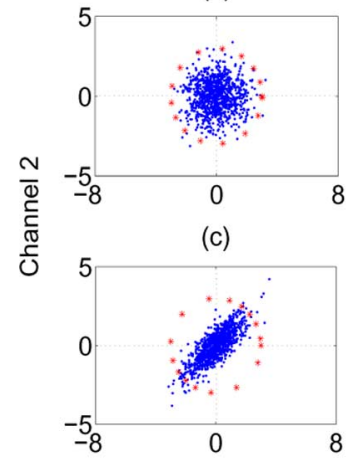

(b)

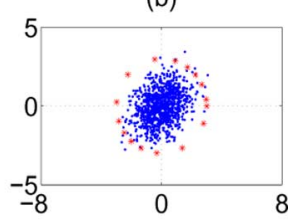

(d)

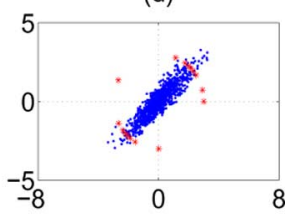

Channel 1

Fig. 2. The scatter plots (blue) and the 16 elliptically distributed projections (red) for a bivariate signal with equal channel powers and varying channel correlations $\eta$. (a) $\eta=0, \epsilon=0.26, \theta=19.45^{\circ}$. (b) $\eta=0.3, \epsilon=0.52, \theta=43^{\circ}$. (c) $\eta=0.7, \epsilon=0.85, \theta=43.4^{\circ}$. (d) $\eta=0.9, \epsilon=0.95, \theta=45^{\circ}$. (a)

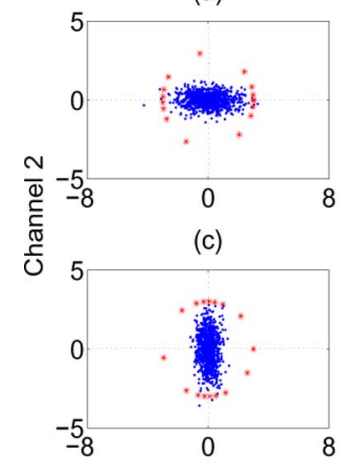

(b)

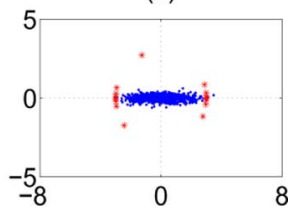

(d)

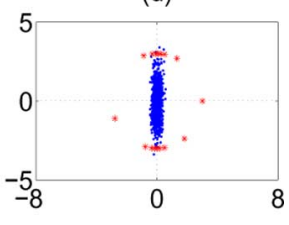

Channel 1

Fig. 3. The scatter plots (blue) and the 16 elliptically distributed projections (red) for an uncorrelated bivariate signal of varying channel power ratio $\Omega$. (a) $\Omega=0.4, \epsilon=0.85, \theta=0.14^{\circ}$. (b) $\Omega=0.16, \epsilon=0.98, \theta=0.04^{\circ}$. (c) $\Omega=2.5, \epsilon=0.85, \theta=89^{\circ}$. (d) $\Omega=6, \epsilon=0.97, \theta=89.7^{\circ}$.

obtained from NS-BEMD for uncorrelated data channels within bivariate signals, with a varying channel power ratio $\Omega$, where $\Omega=\left(\sigma_{\text {channel }_{1}}^{2} / \sigma_{\text {channel }}^{2}\right)$, with $\sigma$ denoting the standard deviation. Notice that, as expected the density of sample points, along the direction of higher power data channels, increases with the discrepancy between channel powers.

\section{Simulation Results}

The performance of the proposed NS-BEMD algorithm was evaluated over comprehensive simulations on bivariate signals with varying degrees of correlation between data channels, varying channel powers, and for a noise-assisted operation of the BEMD for speed estimation from Doppler radar.

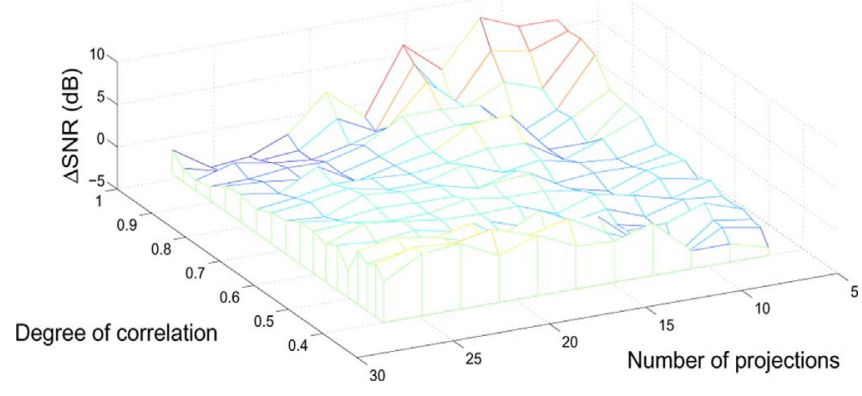

Fig. 4. The difference in reconstruction errors of the IMFs corresponding to tone $s$ in (7), for NS-BEMD and BEMD $(\triangle S N R)$, evaluated against the degree of correlation $\eta$ and the number of projections $k$.

\section{A. Correlated Bivariate Data of Equal Channel Powers}

A single $10 \mathrm{~Hz}$ tone, s, corrupted by white Gaussian noise (WGN), was generated according to:

$$
y_{1}=s+\gamma v_{1} \quad y_{2}=s+\gamma v_{2}
$$

where $v_{1}$ and $v_{2}$ are independent WGN realizations of unit variance. The correlation between $y_{1}$ and $y_{2}$ was governed by the scaling factor $\gamma$. The BEMD and NS-BEMD were applied to the resulting bivariate data $z=\left[y_{1}, y_{2}\right]$ and the reconstruction errors, obtained as the mean square error between the relevant IMF (containing the tone) and the original tone $s$ in (7), were calculated for both BEMD and NS-BEMD. The direction vectors for NS-BEMD were generated as in Fig. 2. Fig. 4 shows the difference between the reconstruction errors (in $\mathrm{dB}$ ) of NS-BEMD and BEMD plotted against the degree of correlation $\eta$ and the number of projections $k$. Notice from Fig. 4 that for high channel correlations (greater than 0.8 ) and for fewer than 20 projections, NS-BEMD greatly outperformed BEMD, whereas it was on par with BEMD when computational complexity was not an issue. For more than 20 projections in Fig. 4 there was no significant difference between NS-BEMD and BEMD even for highly correlated (above 0.8) bivariate signals. This highlights the usefulness of the proposed method for lower numbers of direction vectors (samples), a typical case in real-world applications due to computational constraints.

\section{B. Bivariate Data With Varying Channel Powers}

We next examined the effect of power discrepancy in data channels on the accuracy of NS-BEMD. For one data channel being WGN, we effectively have a noise-assisted decomposition (bivariate way to calculate the standard univariate EMD) [8]. We considered a two tone signal ${ }^{3}$

$$
y=\cos 2 \pi \frac{10}{f_{s}} t+0.11 \cos 2 \pi \frac{30}{f_{s}} t, \quad f_{s}=1000 \mathrm{~Hz}
$$

while the second channel was WGN. By varying the power of the WGN channel relative to that of the signal channel, our aim was to show that the proposed nonuniform sampling scheme caters for the power imbalance. Fig. 5 shows the resulting reconstructed SNRs for both the noise-assisted NSBEMD (NA-NSBEMD) and noise-assisted BEMD (NA-BEMD) algorithms. The best reconstruction of the sinusoids for NA-NSBEMD occurred in the $7 \mathrm{~dB}$ to $9 \mathrm{~dB}$ regions of power imbalance

\footnotetext{
${ }^{3}$ The two tones were selected so that each was in a separate IMF [12].
} 

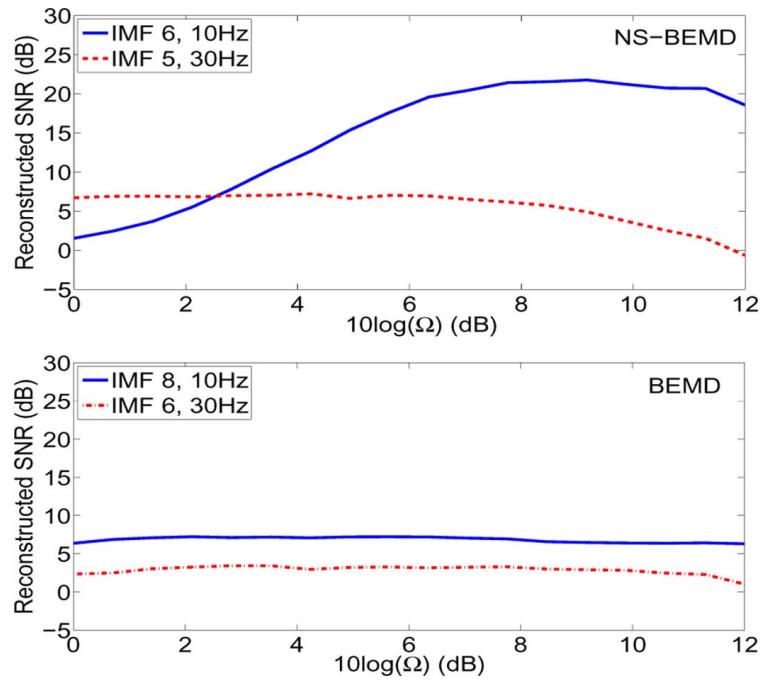

Fig. 5. Reconstruction error (in SNR) in the IMFs for a two-tone signal in (8) with varying channel power ratio, given by $10 \log (\Omega)$. (Top) Reconstruction using of the proposed NA-NSBEMD. (Bottom) Results of NA-BEMD
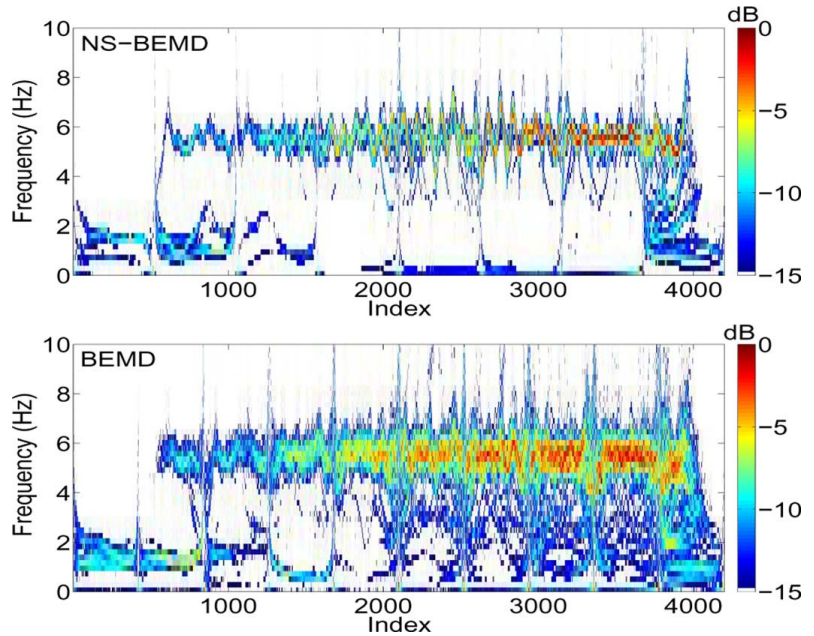

Fig. 6. The time-frequency representation of the Doppler radar signal obtained via NA-NSBEMD (top) and NA-BEMD (bottom) using $k=8$ projections. The noise channel power was $8 \mathrm{~dB}$ relative to the signal $(10 \log (\Omega)=8 \mathrm{~dB})$ ).

$\Omega$, whereas NA-BEMD was not able to account for power imbalance, or to match the performance of NA-NSBEMD.

\section{Speed Estimation Using Doppler Radar}

Fig. 6 shows the time-frequency plot of the IMFs, obtained from decomposing a real-world Doppler radar signature of an object that was moving towards the radar, at a constant speed of $8.3 \mathrm{~cm} / \mathrm{s}$. The corresponding Doppler frequency shift in the radar signal was of $5.5 \mathrm{~Hz}$, and its amplitude linearly increased as the object was traveling towards the radar antenna. To deal with the nonstationarity, the radar signal was segmented into 10 nonoverlapping windows. The time-frequency plots in Fig. 6, show that the noise-assisted NS-BEMD algorithm was able estimate the object speed (evident by a strong frequency signature at around $5.5 \mathrm{~Hz}$ ), together with a better motion localization (increasing spectrogram amplitude as the object approaches the radar).

\section{CONCLUSION}

We have proposed a non-uniform sampling scheme for local mean estimation in bivariate empirical mode decomposition (BEMD), in order to perform projections that adapt to the statistics of the bivariate signals. The proposed algorithm has been shown to be more effective than the original BEMD for correlated channels, and for bivariate data channels with different powers. We have also introduced a way to optimally choose noise levels in noise-assisted versions of BEMD. The proposed algorithm in the noise-assisted framework has been shown to produce a more localized time-frequency representation than the conventional BEMD algorithm, as illustrated by simulations on synthetic and real world data.

\section{APPENDIX}

To show that for a bivariate signal with high noncircularity, the projection samples $\phi_{k}^{\prime}$ are concentrated along the direction $\theta$, from (4), the projection directions

$$
\phi_{k}^{\prime}=\arctan \left(\frac{b}{a} \tan \left(\phi_{k}\right)\right)+\theta .
$$

When $\epsilon \rightarrow 1$, then $(b / a) \rightarrow v$ where $v \ll 1$, and the term $(b / a) \tan \left(\phi_{k}\right) \rightarrow v_{k} \ll 1$. The small angle approximation $\tan (\alpha) \approx \alpha$ results in $\phi_{k}^{\prime}=v_{k}+\theta$, illustrating that the non-uniformly sampled $\phi_{k}^{\prime}$ are concentrated around the direction of principal importance $\theta$. When $\epsilon \rightarrow 0,(b / a) \rightarrow 1$, the term $(b / a) \tan \left(\phi_{k}\right) \approx \tan \left(\phi_{k}\right)$, while (9) becomes $\phi_{k}^{\prime}=\phi_{k}+\theta$, resulting in uniform samples.

\section{REFERENCES}

[1] N. Huang, Z. Shen, S. Long, M. Wu, H. Shih, Q. Zheng, N. Yen, C. Tung, and H. Liu, "The Empirical mode decomposition and the Hilbert spectrum for nonlinear and non-stationary time series analysis," Proc. Roy. Soc A, vol. 454, pp. 903-995, 1998.

[2] T. Tanaka and D. P. Mandic, "Complex Empirical mode decomposition," IEEE Signal Process. Lett., vol. 14, no. 2, pp. 101-104, 2007.

[3] M. U. Altaf, T. Gautama, T. Tanaka, and D. P. Mandic, "Rotation invariant complex empirical mode decomposition," in Proc. IEEE Int. Conf. Acoust., Speech, Signal Process., 2007, vol. 3, pp. 1009-1112.

[4] G. Rilling, P. Flandrin, P. Goncalves, and J. M. Lilly, "Bivariate empirical mode decomposition," IEEE Signal Process. Lett., vol. 14, no. 12, pp. 936-939, 2007.

[5] J. Fleureau, J.-C. Nunes, A. Kachenoura, L. Albera, and L. Senhadji, "Bivariate instantaneous frequency and bandwidth Turning Tangent Empirical mode decomposition: A framework for mono- and multivariate signals," IEEE Trans. Signal Process., vol. 59, no. 3, pp. 1309-1316, 2011.

[6] D. Looney and D. P. Mandic, "Multi-scale image fusion using complex extensions of EMD," IEEE Trans. Signal Process., vol. 57, no. 4, pp. $1626-1630,2009$.

[7] N. Rehman and D. P. Mandic, "Multivariate Empirical mode decomposition," Proc. Roy. Soc. A, vol. 466, no. 2117, pp. 1291-1302, 2010.

[8] N. Rehman and D. P. Mandic, "Filter bank property of multivariate empirical mode decomposition," IEEE Trans. Signal Process., vol. 59, pp. 2421-2426, 2011.

[9] E. Ollila, "On the circularity of a complex random variable," IEEE Signal Process. Lett., vol. 15, pp. 841-844, 2008.

[10] J. M. Lilly and S. C. Olhede, "Bivariate instantaneous frequency and bandwidth," IEEE Trans. Signal Process., vol. 58, no. 2, pp. 591-603, 2010.

[11] E. Ollila, J. Eriksson, and V. Koivunen, "Complex elliptically symmetric random variables - Generation, characterization and circularity tests," IEEE Trans. Signal Process., vol. 59, no. 1, pp. 58-69, 2011.

[12] G. Rilling and P. Flandrin, "One or two frequencies? The Empirical mode decomposition answers," IEEE Trans. Signal Process., vol. 56, no. 1, pp. 85-95, 2008. 